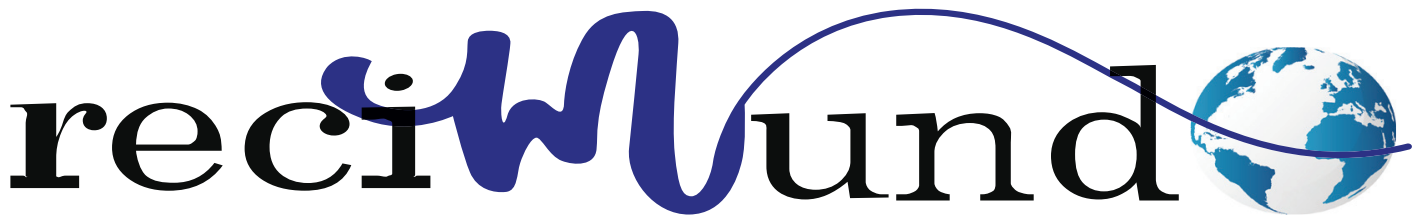

Revista Científica Mundo de la Investigación y el Conocimiento

DOI: 10.26820/recimundo/4.(1).enero.2020.63-70

URL: http://recimundo.com/index.php/es/article/view/747

EDITORIAL: Saberes del Conocimiento

REVISTA: RECIMUNDO

ISSN: 2588-073X

TIPO DE INVESTIGACIÓN: Artículo de Revisión

CÓDIGO UNESCO: 3205 Medicina Interna

PAGINAS: 63-70

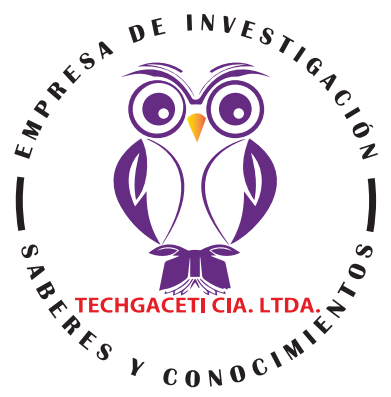

\title{
Complicaciones en la cirugía de hernia inguinal
}

Complications in groin hernia surgery

\section{Complicações na cirurgia de hérnia de virilha}

Sara Alexandra Carbo Yagual'; Francisco Arturo Vaca Morla²; Sandra Dalila Pincay Cevallos;

Clemente Manuel Chang Garcia ${ }^{4}$

RECIBIDO: 18/12/2018 ACEPTADO: 29/03/2019 PUBLICADO: 01/12/2019

1. Médico; Investigador Independiente; Guayaquil, Ecuador; sary_17_85_@hotmail.com; (D) https://orcid. org/0000-0002-0228-7661

2. Médico; Investigador Independiente; Guayaquil, Ecuador; artur800@hotmail.com; (D) https://orcid.org/00000001-5368-6454

3. Médico; Investigador Independiente; Guayaquil, Ecuador, Ecuador; dalilapc13@hotmail.com; (D) https://orcid. org/0000-0001-6237-3396

4. Médico; Investigador Independiente; Guayaquil, Ecuador; clementechang33@gmail.com; (D) https://orcid. org/0000-0001-7413-5926

CORRESPONDENCIA

Sara Alexandra Carbo Yagual

sary_17_85_@hotmail.com

Guayaqui, Ecuador

@ RECIMUNDO; Editorial Saberes del Conocimiento, 2020 


\section{RESUMEN}

Las complicaciones en la cirugía endoscópica de hernia inguinal son más peligrosas y más frecuentes que las de la cirugía abierta, especialmente en manos sin experiencia y, por lo tanto, es mejor evitarlas. Es posible evitar la mayoría de estas complicaciones si uno sigue un conjunto de pasos y principios bien definidos de cirugía de hernia inguinal endoscópica. Se sabe que las complicaciones ocurren en cada paso de la cirugía de hernia. Aplicar precaución al realizar cada paso puede salvar al paciente de mucha morbilidad. Uno comienza aplicando criterios estrictos de selección de pacientes para la reparación de la hernia endoscópica, especialmente en la parte inicial de la curva de aprendizaje. Un conocimiento profundo de la anatomía ayuda mucho a evitar la mayoría de las complicaciones observadas en la reparación de la hernia. Esta anatomía necesita ser reaprendida de lo que uno está acostumbrado, ya que el enfoque es totalmente diferente de una reparación abierta de hernia. Y finalmente, aprender y dominar la técnica correcta es un requisito previo esencial antes de aventurarse en la reparación de la hernia inguinal. Aunque ha habido un aumento en la incidencia de complicaciones reportadas en la reparación endoscópica en las series anteriores, esto puede explicarse en parte por el hecho de que estaba en la primera parte de la curva de aprendizaje de la mayoría de los cirujanos endoscópicos. A medida que la experiencia creció y las técnicas se estandarizaron, la incidencia de complicaciones también se redujo y estuvo a la par con la cirugía abierta de hernia. Se discutirán las diversas complicaciones y precauciones que se deben tomar para evitarlas.

Palabras clave: Endoscópico, Hernia inguinal, Complicaciones.

\section{ABSTRACT}

Complications in endoscopic inguinal hernia surgery are more dangerous and more frequent than those of open surgery, especially in inexperienced hands and hence are best avoided. It is possible to avoid most of these complications if one follows a set of well-defined steps and principles of endoscopic inguinal hernia surgery. Complications are known to occur at each and every step of hernia surgery. Applying caution while performing each step can save the patient from a lot of morbidity. One starts by applying strict patient selection criteria for endoscopic hernia repair, especially in the initial part of ones learning curve. A thorough knowledge of anatomy goes a long way in avoiding most of the complications seen in hernia repair. This anatomy needs to be relearned from what one is used to, as the approach is totally different from an open hernia repair. And finally, learning and mastering the right technique is an essential prerequisite before one venture into inguinal hernia repair. Although there has been an increased incidence of complications reported in endoscopic repair in the earlier series, this can be explained partly by the fact that it was in the early part of the learning curve of most endoscopic surgeons. As the experience grew and the techniques were standardized, the incidences of complications have also reduced and have come to be on par with open hernia surgery. The various complications and precautions to be taken to avoid them will be discussed.

Keywords: Endoscopic, Inguinal hernia, Complications.

\section{RESUMO}

As complicações na cirurgia endoscópica da hérnia inguinal são mais perigosas e mais frequentes do que as da cirurgia aberta, especialmente em mãos inexperientes e, portanto, devem ser evitadas. É possível evitar a maioria dessas complicações se seguirmos um conjunto de etapas e princípios bem definidos da cirurgia endoscópica de hérnia inguinal. Sabe-se que as complicações ocorrem em cada etapa da cirurgia de hérnia. A cautela ao executar cada etapa pode salvar o paciente de muita morbidade. Começa-se aplicando critérios rigorosos de seleção de pacientes para correção de hérnia endoscópica, principalmente na parte inicial da curva de aprendizado. Um conhecimento aprofundado da anatomia ajuda bastante a evitar a maioria das complicações vistas no reparo da hérnia. Essa anatomia precisa ser reaprendida a partir do que estamos acostumados, pois a abordagem é totalmente diferente de um reparo aberto de hérnia. E, finalmente, aprender e dominar a técnica correta é um pré-requisito essencial antes de se aventurar no reparo da hérnia inguinal. Embora tenha havido um aumento na incidência de complicações relatadas no reparo endoscópico nas séries anteriores, isso pode ser explicado em parte pelo fato de estar na parte inicial da curva de aprendizado da maioria dos cirurgiões endoscópicos. À medida que a experiência cresceu e as técnicas foram padronizadas, a incidência de complicações também diminuiu e passou a ser comparável à cirurgia de hérnia aberta. As várias complicações e precauções a serem tomadas para evitá-las serão discutidas.

Palavras-chave: Endoscópica, Hérnia inguinal, Complicações. 


\section{Introducción}

Una hernia inguinal es una condición en la cual grasa intraabdominal o parte del pequeño intestino, también llamado intestino delgado, crea protuberancias a través de un área débil en la parte inferior del abdomen músculos. Se produce una hernia inguinal en la ingle: el área entre el abdomen y muslo. Este tipo de hernia se llama inguinal porque la grasa o parte del intestino se desliza a través de un área débil en el anillo inguinal, la apertura al canal inguinal. Una hernia inguinal aparece como una protuberancia en uno o ambos lados de la ingle. Una hernia inguinal puede ocurrir en cualquier momento desde la infancia hasta la edad adulta y es mucho más común en varones que hembras. Las hernias inguinales tienden a convertirse más grande con el tiempo.

Cada año, muchos son los pacientes que se someten a una reparación de hernia inguinal. Es una de las operaciones más comunes realizadas en hospitales. Hay una tasa estimada de complicaciones del diez por ciento asociado con el procedimiento (Nyhus, Artz, \& Hardy, 2012). Las complicaciones postoperatorias siempre son motivo de preocupación para el cirujano. Algunos son de poca importancia, otros conducen a una discapacidad prolongada, y algunos terminan en la muerte. Es del cirujano la responsabilidad de garantizar su prevención, reconocimiento y gestión.

Cuatro categorías de complicaciones existen. Ellas son:

(1) Los que surgen de la enfermedad por la cual es la operación realizado

(2) Las derivadas de afecciones asociadas no relacionadas con el trastorno primario

(3) Las derivadas directamente del procedimiento quirúrgico en sí

(4) Las debidas en parte a cualquiera de los grupos anteriores, pero no imputables a ninguno.
Es el propósito de esta discusión para enfatizar algunos de las complicaciones más comunes de Reparación de hernia inguinal.

La observación cuidadosa de ciertas características en la técnica quirúrgica puede prevenir muchas de las complicaciones. Estas características incluyen: (1) disección limpia y afilada (2) evitación de disección áspera y trauma a partir de una gasa o dedos, (3) uso de volúmenes mínimos de agentes anestésicos locales, (4) uso apropiado de incisiones relajantes para evitar tensiones, (5) utilización de suturas no absorbibles, es decir, tipo monofilamento si existe potencial de contaminación, y (6) irrigación de cada capa de la herida.

\section{Metodología}

Para el desarrollo de este proceso investigativo, se plantea como metodología la encaminada hacia una orientación científica particular que se encuentra determinada por la necesidad de indagar en forma precisa y coherente una situación, en tal sentido (Davila, 2015) define la metodología "como aquellos pasos previos que son seleccionados por el investigador para lograr resultados favorables que le ayuden a plantear nuevas ideas".(p.66)

Lo citado por el autor, lleva a entender que el desarrollo de la acción investigativa busca simplemente coordinar acciones enmarcadas en una revisión bibliográfica con el fin de complementar ideas previas relacionadas a las complicaciones de la cirugía de hernia inguinal a través de una revisión de literatura, para así finalmente elaborar un cuerpo de consideraciones generales que ayuden a ampliar el interés propuesto.

\section{Tipo de Investigación}

Dentro de toda práctica investigativa, se precisan acciones de carácter metodológico mediante las cuales, se logra conocer y proyectar los eventos posibles que la determinan, así como las características que hacen del acto científico un proceso interac- 
tivo ajustado a una realidad posible de ser interpretada. En este sentido, se puede decir, que la presente investigación corresponde al tipo documental, definido por Castro (2016), "se ocupa del estudio de problemas planteados a nivel teórico, la información requerida para abordarlos se encuentra básicamente en materiales impresos, audiovisuales y /o electrónicos". (p.41).

En consideración a esta definición, la orientación metodológica permitió la oportunidad de cumplir con una serie de actividades inherentes a la revisión y lectura de diversos documentos donde se encontraron ideas explicitas relacionadas con los tópicos encargados de identificar a cada característica insertada en el estudio. Por lo tanto, se realizaron continuas interpretaciones con el claro propósito de revisar aquellas apreciaciones o investigaciones propuestas por diferentes investigadores relacionadas con el tema de interés, para luego dar la respectiva argumentación a los planteamientos, en función a las necesidades encontradas en la indagación.

\section{Fuentes Documentales}

El análisis correspondiente a las características que predomina en el tema seleccionado, llevan a incluir diferentes fuentes documentales encargadas de darle el respectivo apoyo y en ese sentido cumplir con la valoración de los hechos a fin de generar nuevos criterios que sirven de referencia a otros procesos investigativos. Para (CASTRO, 2016) las fuentes documentales incorporadas en la investigación documental o bibliográfica, "representa la suma de materiales sistemáticos que son revisados en forma rigurosa y profunda para llegar a un análisis del fenómeno".(p.41). Por lo tanto, se procedió a cumplir con la realización de una lectura previa determinada para encontrar aquellos aspectos estrechamente vinculados con el tema, con el fin de explicar mediante un desarrollo las respectivas apreciaciones generales de importancia.

\section{Técnicas para la Recolección de la Infor- mación}

La conducción de la investigación para ser realizada en función a las particularidades que determinan a los estudios documentales, tiene como fin el desarrollo de un conjunto de acciones encargadas de llevar a la selección de técnicas estrechamente vinculadas con las características del estudio. En tal sentido, (Bolívar, 2015), refiere, que es "una técnica particular para aportar ayuda a los procedimientos de selección de las ideas primarias y secundarias". (p. 71).

Por ello, se procedió a la utilización del subrayado, resúmenes, fichaje, como parte básica para la revisión y selección de los documentos que presentan el contenido teórico. Es decir, que mediante la aplicación de estas técnicas se pudo llegar a recoger informaciones en cuanto a la revisión bibliográfica de los diversos elementos encargados de orientar el proceso de investigación. Tal como lo expresa, (Bolívar, 2015) "las técnicas documentales proporcionan las herramientas esenciales y determinantes para responder a los objetivos formulados y llegar a resultados efectivos" (p. 58). Es decir, para responder con eficiencia a las necesidades investigativas, se introdujeron como técnica de recolección el método inductivo, que hizo posible llevar a cabo una valoración de los hechos de forma particular para llegar a la explicación desde una visión general.

Asimismo, se emplearon las técnicas de análisis de información para la realización de la investigación que fue ejecutada bajo la dinámica de aplicar diversos elementos encargados de determinar el camino a recorrer por el estudio, según, (Bolívar, 2015) las técnicas de procesamiento de datos en los estudios documentales "son las encargadas de ofrecer al investigador la visión o pasos que debe cumplir durante su ejercicio, cada una de ellas debe estar en correspondencia con el nivel a emplear" (p. 123). Esto indica, que para llevar a cabo el pro- 
cesamiento de los datos obtenidos una vez aplicado las técnicas seleccionadas, tales como: fichas de resumen, textual, registros descriptivos entre otros, los mismos se deben ajustar al nivel que ha sido seleccionado.

\section{Resultados}

Las complicaciones si se siguen un conjunto de pasos y principios bien definidos de cirugía laparoscópica. La incidencia de complicaciones ha disminuido a medida que la experiencia ha crecido y ha demostrado ser un procedimiento seguro en manos de cirujanos experimentados.

Aquí discutiremos varias complicaciones, así como los pasos y precauciones que se deben tomar para evitarlos.

Las complicaciones y las diversas precauciones que se deben tomar en la cirugía de hernia se pueden dividir en:

1) Preoperatorio

2) Intraoperatorio

3) Postoperatorio

\section{Precauciones preoperatorias}

La precaución preoperatoria más importante es la selección adecuada del paciente antes de la cirugía, especialmente en la parte inicial de la curva de aprendizaje. Idealmente, las hernias indirectas, directas o pequeñas son las mejores. Las hernias grandes, los pacientes obesos y las hernias irreductibles y obstruidas se evitan mejor. Una contraindicación absoluta es la hernia estrangulada. También es obligatorio un estudio detallado de pacientes de edad avanzada para evaluar el estado cardiorrespiratorio para garantizar un resultado seguro.

\section{Complicaciones y precauciones intraope- rativas}

En manos inexpertas, las complicaciones en la cirugía laparoscópica de hernia inguinal son más peligrosas y más frecuentes que las de la cirugía abierta y, por lo tanto, es mejor evitarlas. Es posible evitar la mayoría de estos:

\section{1) Durante la creación del espacio prepe- ritoneal. Este es el paso más importante para los principiantes.}

- Una amplia línea alba puede ocasionar la ruptura del peritoneo; En tal situación, es mejor cerrar el recto e incidir la vaina más lateralmente

- Colocación inadecuada del trocar con globo que causa la disección de las fibras musculares

- Entrada al peritoneo que causa neumoperitoneo

- Ruptura del globo en el espacio preperitoneal

- El trocar de Hassan debe encajar perfectamente en la incisión para evitar fugas de $\mathrm{CO} 2$

Para evitar esto, debe asegurarse de que el globo esté hecho correctamente y se ingrese al espacio correcto retrayendo el músculo recto lateralmente para visualizar la vaina posterior del recto. Además, el trócar con globo se inserta suavemente, paralelo a la pared abdominal, para evitar perforar el peritoneo. El globo debe inflarse lentamente con solución salina para garantizar una distensión suave y uniforme y evitar su ruptura.

\section{2) Precauciones durante la colocación del puerto}

Los trócares deben ser cortos y roscados en proporción a menos espacio de trabajo y para garantizar un ajuste perfecto, respectivamente. Las incisiones en la piel deben ser adecuadas para agarrar el trocar y evitar su deslizamiento. El paciente debe vaciar su vejiga antes de la cirugía, ya que el trocar suprapúbico podría dañar una vejiga llena. La presión en el espacio preperitoneal debe ser tal que ofrezca resistencia suficiente durante la inserción del trocar para evitar perforar el peritoneo. 


\section{3) Identificación correcta de los puntos de referencia anatómicos.}

El siguiente paso más importante y crucial en cualquier cirugía de hernia es la identificación correcta de puntos de referencia anatómicos. Esto es difícil para los principiantes ya que la anatomía es diferente de la observada en la cirugía abierta. El primer paso más importante es identificar el hueso púbico. Una vez que esto se ve, el resto de los puntos de referencia se rastrean manteniendo esto como punto de referencia. Se recomienda mantenerse alejado del triángulo de la fatalidad, que contiene los vasos ilíacos y evitar colocar tachuelas en el triángulo del dolor lateralmente.

\section{4) Lesión de la vejiga}

La lesión de la vejiga ocurre con mayor frecuencia durante la colocación del puerto, diseccionando un saco directo grande o en una hernia deslizante. Es obligatorio vaciar la vejiga antes de una reparación de hernia inguinal para evitar una lesión en el trocar. Es aconsejable que los principiantes catetericen la vejiga durante la parte inicial de su curva de aprendizaje. El diagnóstico es evidente cuando se ve orina en el espacio extraperitoneal. La reparación se realiza con vicryl en dos capas y un catéter urinario insertado durante 7-10 días.

\section{5) Lesión intestinal}

Es raro durante la cirugía de hernia. Puede ocurrir cuando se reducen las hernias grandes, la apertura involuntaria del peritoneo hace que el intestino ingrese al campo de la cirugía y en la reducción de las hernias deslizantes. Es mejor evitar las lesiones en tales circunstancias abriendo el saco herniario lo más cerca posible del anillo profundo. Los estudios iniciales (Grant, 2012) mostraron una mayor incidencia, especialmente con TAPP, pero disminuyó con el tiempo.

\section{6) Lesión vascular}

Esta es una de las lesiones más comunes que ocurren en la reparación de la hernia y, a menudo, una razón para la conversión. Los diversos sitios donde puede ocurrir es la lesión de los vasos del músculo recto durante la inserción del trocar; lesión del vaso epigástrico inferior; sangrado del plexo venoso en la sínfisis púbica; lesión de la vena obturadora aberrante; lesión del vaso testicular; y el más desastroso de todos, los vasos ilíacos, que requieren una conversión de emergencia para controlar el sangrado y los servicios inmediatos de un cirujano vascular para repararlos. La mayoría de las otras hemorragias se pueden controlar con cauterización o clips. La disección cuidadosa y el cumplimiento de los principios de la cirugía ayudarán a evitar la mayoría de estas lesiones.

\section{7) Lesión a los conductos deferentes}

La lesión ocurre mientras se diseca el saco herniario de las estructuras del cordón. La lesión provoca un eventual estrechamiento fibrótico del conducto. Una transección completa del conducto debe repararse en un paciente joven. Es mejor evitar una lesión en el conducto vascular y esto se puede hacer identificando antes de dividir cualquier estructura cerca del anillo profundo o el piso del espacio extraperitoneal. Además, la separación de las estructuras del cordón del saco herniario debe ser suave y directa; Se debe evitar el agarre de los conductos deferentes con fórceps.

\section{8) Neumoperitoneo}

Es una ocurrencia común en TEP que todo cirujano debe estar preparado para manejar. Poner al paciente en posición de Trendelenberg y aumentar las presiones de insuflación a $15 \mathrm{mmHg}$ ayuda. Si el problema persiste, se puede insertar una aguja Veress en el punto de Palmer.

\section{Complicaciones postoperatorias}

\section{1) Formación de seroma / hematoma}

Es una complicación común después de una cirugía de hernia laparoscópica, cuya incidencia está en el rango de 5-25\%. Se 
ven especialmente después de la reparación indirecta de una gran hernia. La mayoría se resuelve espontáneamente durante 4-6 semanas. Se puede evitar un seroma minimizando la disección del saco herniario de las estructuras del cordón, fijando el saco directo al hueso púbico y fenestrando la fascia transversal en una hernia directa. Algunos cirujanos ponen un drenaje si hay sangrado excesivo o después de una disección extensa.

\section{2) Retención urinaria}

Esta complicación después de la reparación de la hernia tiene una incidencia informada de 1.3 a 5.8\% (Fitzgibbons, y otros, 2005) Por lo general, se precipita en pacientes de edad avanzada, especialmente si hay síntomas de prostatismo. Es mejor cateterizar a estos pacientes antes de la cirugía y retirar el catéter al día siguiente por la mañana.

\section{3) Neuralgias}

Se informa que la incidencia de esta complicación es de entre 0.5 y $4.6 \%$ dependiendo de la técnica de reparación. El método de malla de recubrimiento intraperitoneal tuvo la mayor incidencia de neuralgias en un estudio y, por lo tanto, se abandonó como una forma de reparación viable (Fitzgibbons, y otros, 2005). Los nervios comúnmente afectados son el nervio cutáneo lateral del muslo, el nervio genitofemoral y el nervio cutáneo intermedio del muslo. Por lo general, están involucrados por fibrosis inducida por malla o atrapamiento por una tachuela. La complicación se evita evitando fijar la malla lateral al anillo inguinal profundo en la región del triángulo del dolor, la disección segura de un gran saco herniario y la no disección de la fascia sobre el psoas.

\section{4) Dolor e hinchazón testicular}

Ocurre debido a la disección excesiva de un saco de las estructuras del cordón, especialmente un saco completo. La incidencia reportada es de 0.9 a 1.5\%. La mayoría son transitorios. Se encontró orquitis en un pequeño número de pacientes (Phillips, y otros, 2005) pero no condujo a atrofia testicular.

\section{5) Infección de malla e infección de la he- rida}

Las tasas de infección de la herida son muy bajas. La infección de la malla es una complicación muy grave y se debe tener cuidado para mantener precauciones asépticas estrictas durante todo el procedimiento. Cualquier infección endógena debe tratarse con un tratamiento adecuado de antibióticos antes de la cirugía.

\section{6) recurrencia}

Es el punto final más importante de cualquier cirugía de hernia. Requiere un conocimiento adecuado y completo de la anatomía y una técnica completa de reparación para ayudar a mantener la recurrencia en la reparación endoscópica al mínimo.

Los puntos importantes a tener en cuenta durante la cirugía son:

- Después de diseccionar el saco directo, todas las adherencias peritoneales alrededor del margen del defecto se deben lisar meticulosamente.

- Siempre busque un saco indirecto, incluso si se ha reducido una hernia directa.

- Refleje el peritoneo del cordón completamente.

- Coloque una malla de tamaño adecuado para cubrir completamente el orificio miopectineal, preferiblemente del tamaño de $15 \times 15 \mathrm{~cm}$.

- El margen inferior de la malla debe colocarse cómodamente, medialmente en el espacio retropúbico y lateralmente sobre el músculo psoas.

- Realice una fijación de 2 puntos de la malla en el aspecto medial sobre el ligamento de Cooper.

- Evite cortar la malla sobre el cordón.

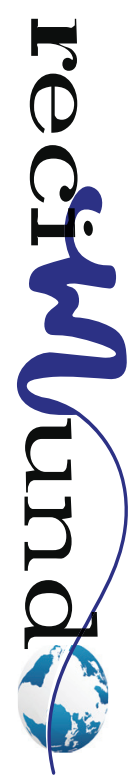


Esto debilita la malla y proporciona un sitio potencial para la recurrencia.

- Asegure una hemostasia adecuada antes de colocar la malla.

- El factor más importante es la capacitación y el aprendizaje adecuados de la técnica correcta.

\section{Conclusiones}

La reparación endoscópica de hernia es una técnica que pronto se convertirá en una parte importante del arsenal de un cirujano de hernia. Aunque la incidencia inicial de complicaciones después de la reparación laparoscópica de la hernia fue mayor, ha disminuido después de la estandarización de la técnica. (Felix, Harbertson, \& Vartanian, 2009) encontraron una tasa de complicaciones del $6 \%$ durante un período de 6 años, pero cuando se dividió en dos períodos de 3 años, su tasa fue del 3,6\% en los primeros 3 años y cayó al 0,5\% en los siguientes 3 años. La mayoría de los estudios a largo plazo han mostrado una baja incidencia de morbilidad después de una reparación endoscópica. Al seleccionar adecuadamente a un paciente y tener un conocimiento profundo de la anatomía y una experiencia adecuada, se puede realizar de forma segura la reparación endoscópica manteniendo las complicaciones al mínimo.

\section{Bibliografía}

Bolívar, J. (2015). Investigación Documental. México: Pax.

Castro, J. (2016). Técnicas Documentales. México: Limusa.

Davila, A. (2015). Concepto de terminos cientificos. Caracas: Oasis.

Felix, E., Harbertson, N., \& Vartanian, S. (2009). Laparoscopic hernioplasty: Significant complications. Surg Endosc, 13(4), 328-31. Obtenido de https://www.ncbi.nlm.nih.gov/pubmed/10094740

Fitzgibbons, R. J., Camps, J., Cornet, D., Nguyen, N., Litke, B., \& Annibali, R. (2005). Laparoscopic inguinal herniorrhaphy. Results of multicenter trial. Ann Surg, 221(1), 3-13. Obtenido de https://www. ncbi.nlm.nih.gov/pubmed/7826159

Grant, A. (2012). EU Hernia Trialists Collaboration. Laparoscopic versus open groin hernia repair: Meta-analysis of randomised trials based on individual patient data. Hernia, 6(1), 2-10. Obtenido de https://www.ncbi.nlm.nih.gov/pubmed/12090575

Nyhus, L., Artz, C., \& Hardy, J. (2012). Complications of hernial repair. Management of Surgical Complications. Philadelphia: WB Saunders.

Phillips, E., Arregui, M., Carroll, B., J, C., WB, C., \& Fallas, M. (2005). Incidence of complications following laparoscopic hernioplasty. Surg Endosc, 9(1), 16-21. Obtenido de https://www.ncbi.nlm.nih. gov/pubmed/7725207

\section{CITAR ESTE ARTICULO:}

Carbo Yagual, S., Vaca Morla, F., Pincay Cevallos, S., \& Chang Garcia, C. (2020). Complicaciones en la cirugía de hernia inguinal. RECIMUNDO, 4(1), 63-70. doi:10.26820/recimundo/4.(1).enero.2020.63-70

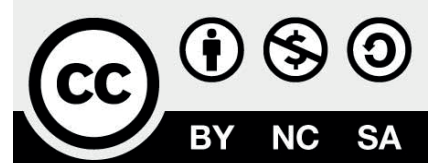

RECONOCIMIENTO-NOCOMERCIAL-COMPARTIRIGUAL CC BY-NC-SA

ESTA LICENCIA PERMITE A OTROS ENTREMEZCLAR, AJUSTARY CONSTRUIR A PARTIR DE SU OBRA CON FINES NO COMERCIALES, SIEMPRE Y CUANDO LE RECONOZCAN LA AUTORÍA Y SUS NUEVAS CREACIONES ESTÉN BAJO UNA LICENCIA CON LOS MISMOS TÉRMINOS. 